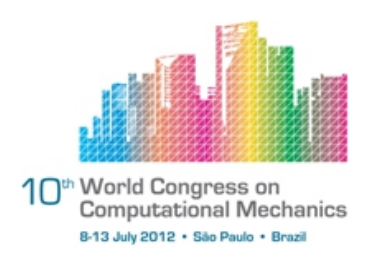

\title{
CONSTITUTIVE MODELING OF PRESTRESSED SHAPE MEMORY FIBER-MATRIX COMPOUNDS
}

\author{
B. Kohlhaas ${ }^{1}$, S. Klinkel ${ }^{1}$ \\ ${ }^{1}$ Institute of Structural Mechanics, TU Kaiserslautern University \\ Paul-Ehrlich-Str. 14, 67663 Kaiserslautern, Germany \\ (benedikt.kohlhaas@bauing.uni-kl.de, sven.klinkel@bauing.uni-kl.de)
}

Abstract. This contribution is concerned with a constitutive model for shape memory alloy

(SMA) fibers. The model accounts for all material phenomena. It incorporates pseudoplasticity and the shape memory effect (SME). These phenomena occur in the low temperature range. For high temperature phase, the pseudoelastic behavior occurs. Additionally, the constrained SME (CSME) and the two-way SME are captured by the model. With respect to the assumption of small strains an additive split of the strain in an elastic and inelastic part is suggested. A free energy function is defined as a function of the elastic strain and an internal hardening variable. The constitutive equations for the stress and the conjugate hardening variable are derived from free energy. The elastic range is defined by two yield criteria, which are similar to kinematic hardening, known from classical plasticity theory. Here, the cases of loading and unloading are distinguished. These criteria are functions of the stress, the conjugate hardening variable and the energy difference between the austenitic and the martensitic phase, the so-called driving variable. The evolution equations for the inelastic state variables, namely the inelastic strain, the internal hardening variable and an inner variable, describing the martensitic volume evolution, are in accordance with the second law of thermodynamics. They are derived from the principle of maximum dissipation with the yield criteria as constraint. Following standard arguments, the martensite volume fraction is decomposed into twinned and oriented martensite. The first one is able to transform into austenite due to heating and vice versa. Just as well, it can change into the second one applying mechanical stress. The constitutive model is embedded in a one-dimensional truss formulation and implemented into a finite element analysis program. Numerical examples show the capability of the formulation. Simulations demonstrate the wide range of possible applications of SMA. A fiber-matrix composite is discussed, which allows for prestressing structures. This necessitates a precise description of the CSME. Likewise, this effect is used to prestress a representative volume element.

Keywords: shape memory fiber, composite, pseudoelasticity, pseudoplasticity, shape memory effect 


\section{INTRODUCTION}

The potential lying in shape memory alloys is already known for quite a while. In the past 30 years a lot of effort was done to capture the material behavior, describe it mathematically and to investigate possible applications. Because of the huge community of researchers it is not possible to mention every single work. The following paragraph gives an overview on important publications, but doesn't claim completeness.

A wide range of applications of shape memory alloys (SMA) may be found in mechanical, robotic and biomechanical engineering, but also in civil engineering promising applications are possible. One aspect are the good damping properties for earthquake engineering or vibration control (i.e. McCormick et al. [23], Castellano et al. [7]). Other authors use shape memory alloys for additional reinforcement in masonry structures (see Indirli et al. [16], [17]) or self-healing applications [29]. A new proposal for the use of shape memory alloys was presented in Moser et al. [24] where the material is introduced as fiber-reinforcement in concrete structures. The possibility of prestressing those structures subsequently is discussed. An overview on possible applications in civil engineering is given in the works of Janke et al. [18], Klinkel and Kohlhaas [20] and Song et al. [29].

A lot of publications may be found which are concerned with the modeling and computational analysis of shape memory alloys. An early publication is made by Achenbach and Müller [1]. Classical tools of statistical mechanics are used to represent the stress-strain-behavior at different temperatures. Achenbach's thoughts were enhanced by Seelecke and Müller [27]. Brinson [6] was the first one who introduced an additive split for the martensite-volumefraction. His 1-D model was based on Helmholtz' free energy function. This idea was picked up by Leclercq and Lexcellent [22]. They use exponential hardening functions and are able to describe pseudoelasticity and pseudoplasticity for the 3-D case. Another work of Lexcellent in cooperation with Raniecki [26] is able to model the tension-compression asymmetry. The model is derived in contradiction to the before mentioned works on basis of Gibb's free energy. Bo and Lagoudas [2], [3], [4], [5] refine the model in a series of papers and take plasticity into account. Helm and Haupt [12], [13], [14], [15] formulate a phenomenological 3-D material model in the framework of small and large strains on basis of Helmholtz' free energy function. The model captures the rate-dependence of shape memory alloys. It can display pseudoplasticity, pseudoelasticity as the memory effects. The finite element implementation was done for pseudoelasticity. Christ and Reese [8], [9], [10] improve the model of Helm and Haupt. They implement each material phenomenon into the finite element method. A reduced integration algorithm in combination with an Hourglas stabilization is used. A good overview on existent works and further research topics on shape memory alloys is given in Lagoudas [21].

But, the more complex the material models get and the more material phenomena are captured, the more calculation time is needed for simulations. In addition, it becomes even more difficult to discretize a structure with a detailed microstructure. With respect to application in civil engineering, like sma-fiber reinforced concrete, the presented formulation is restricted to small strain theory which is sufficient to capture the essential effects. This contribution aims for a fast, robust and simple algorithm which captures the most important material phenomena like pseudoplasticity, pseudoelasticity as the shape memory effect, the constraint shape 
memory effect and the two-way effect. The material model is incorporated in a structural element, which should be capable of easy meshing with respect to fiber-composite structures. So, a material model is presented which is based on the works of Helm [12] and Christ [9]. In order of a reduced computational effort, it is reduced to a fast 1-D algorithm neglecting the rate-dependency which is dispensable for this purpose. A reduction of a 3-D model down to a beam formulation was formerly performed by Evangelista in [11].

The main features of the present formulation are:

i A thermodynamic consistent model is presented which is based on the second law of thermodynamics.

ii A simplified free energy function is used. On its basis, the constitutive equations are derived.

iii The model captures the pseudoplastic state, the pseudoelastic state and arbitrary intermediate states can be displayed properly.

The model is implemented into a truss element. The ability of the formulation to represent the relevant material phenomena will be shown in numerical examples. In addition, the examples will put emphasize on the good use in composite structures. A combination of shape memory alloy fibers with other materials enhances the range of possible applications. A new family of smart compound-structures like fiber-reinforced concrete or grip-elements become possible.

\section{A Simple, Constitutive Material Model For Shape Memory Alloy Fibers}

\subsection{Kinematics}

To model shape memory fibers a one-dimensional model is introduced. For the assumption of small deformations the geometric field equation reduces to

$$
\varepsilon=\frac{\partial u}{\partial x}=u^{\prime}
$$

Due to the fact that for applications in civil engineering, e.g. shape memory fiber reinforced concrete, only small deformations occur small strains

$$
\varepsilon=\varepsilon^{e}+\varepsilon^{i}
$$

are assumed.

\subsection{Free Energy Equation}

The material model is derived on the basis of a free Helmholtz energy function. A certain form for this energy function

$$
\psi=z \cdot \psi^{M}+(1-z) \cdot \psi^{A}
$$

is proposed in [13]. Here, the overall free energy is additively decomposed in a free energy part for the martensitic and the austenitic fraction. The amount of each phase is controlled via 
the martensite volume fraction $z$. The latter one is of scalar type and takes values between zero and one $(0 \leq z \leq 1)$. The martensitic and the austenitic energy part are given as

$$
\begin{aligned}
\psi^{A / M} & =\psi^{A / M}\left(\varepsilon^{e}, \alpha, \theta, z\right) \\
& =\frac{1}{2} \varepsilon^{e} E^{A / M} \varepsilon^{e}+\frac{1}{2} \alpha K^{A / M} \alpha+c_{d}^{A / M}\left(\theta-\theta_{0}\right)+u_{0}^{A / M}-\theta\left[c_{d}^{A / M} \ln \frac{\theta}{\theta_{0}}+\eta_{0}^{A / M}\right]
\end{aligned}
$$

and have the same form. It consists of an elastic, an inelastic and a calorian energy part. Assuming the same material parameters for each phase and inserting equation (4) into equation (3) yields a simplified free energy function

$$
\psi=\frac{1}{2} \varepsilon^{e} E \varepsilon^{e}+\frac{1}{2} \alpha K \alpha+z \cdot\left(\Delta u_{0}-\theta \Delta \eta_{0}\right)-\theta \cdot\left[c_{d} \ln \frac{\theta}{\theta_{0}}+\eta_{0}^{A}-c_{d}\right]-c_{d} \theta_{0}+u_{0}^{A} .
$$

In this equation the elastic energy part can be recognized by the use of the young's modulus $E$ and the elastic strain $\varepsilon^{e}$. Further, the inelastic energy part incorporates the hardening modulus $K$ and the inelastic, inner variable $\alpha$. The last term to be found in equation (5) is a calorian function. In addition to the martensite volume fraction $z$ the temperature $\theta$ and the effective heat capacity $c_{d}$ occur. The variable $u_{0}$ stands for the inner energy and $\eta_{0}$ for the entropy in unloaded conditions. $\Delta$ indicates the difference of the inner energy $u$ respectively the entropy $\eta$ between the austenitic and the martensitic phase.

\subsection{Constitutive Equations}

The constitutive equations are derived by taking the partial derivative of the free energy function with respect to the inner variables.

The stress $\sigma$ is defined as

$$
\sigma=\frac{\partial \psi}{\partial \varepsilon^{e}}=E \cdot \varepsilon^{e}=E \cdot\left(\varepsilon-\varepsilon^{i}\right)
$$

In the same way, a quantity $p$ is derived by

$$
p=-\frac{\partial \psi}{\partial \alpha}=-K \cdot \alpha .
$$

This quantity is also of stress type. In comparison to classical plasticity, $p$ is similar to a backstress, known from kinematic hardening. At least, the derivation with respect to the martensitic volume fraction $z$ reads

$$
\Delta \psi=\Delta \psi(\theta)=\frac{\partial \psi}{\partial z}=\Delta u_{0}-\theta \Delta \eta_{0} .
$$

$\Delta \psi$ is very important for the phase transition of the presented model. In literature it is referred to as driving force ([8], [9]) or thermodynamic force ([14], [15]), for further explanation see section 2.4 .

\subsection{Phase Transition Conditions}

Classical plasticity theory uses one yield condition to differentiate between the elastic and plastic range [28]. The elastic range defines the set of the admissible stresses. Shape 
memory alloys provide a more complex stress-strain-behavior. One yield condition, phase transition condition, respectively is no more sufficient. Here, two conditions of that kind are used. To distinguish between loading and unloading the following criterion is used

$$
\stackrel{\leftrightarrow}{\varepsilon}=\varepsilon^{i} \cdot \operatorname{sign}\left(\sigma-p-\frac{\langle\Delta \psi\rangle}{\beta} \cdot \operatorname{sign}\left(\varepsilon^{i}\right)\right)=\left\{\begin{array}{l}
\geq 0, \text { loading } \\
<0, \text { unloading }
\end{array}\right.
$$

The Macaulay-bracket $\langle\ldots\rangle$ is defined as $\langle x\rangle=(|x|+x) / 2$. The case of loading is bonded by

$$
\vec{\Phi}(\sigma, p, \Delta \psi)=\left|\sigma-p-\frac{\langle\Delta \psi\rangle}{\beta} \cdot \operatorname{sign}(\sigma-p)\right|-\sigma_{\mathrm{y}} \leq 0
$$

The description of phase transitions in the case of loading necessitates a stress criterion which is given as

$$
\sigma \stackrel{!}{\geq} \sigma_{\mathrm{ctrl}}=|\sigma-p|-\frac{\langle\Delta \psi\rangle}{\beta} .
$$

Unloading the material the following phase transition equation

$$
\overleftarrow{\Phi}(\sigma, p, \Delta \psi)=\left|\sigma-p-\frac{\langle\Delta \psi\rangle}{\beta} \cdot \operatorname{sign}\left(\varepsilon^{i}\right)\right|-\sigma_{\mathrm{y}} \leq 0
$$

limits the elastic range. In equations (9) $-(12) \sigma_{\mathrm{y}}$ and $\beta$ are material parameters. $\sigma_{\mathrm{y}}$ measures half the height of the hysteresis and $\beta$ indicates the maximum hysteresis width. The quantity $\Delta \psi$ works like an offset (compare [12]). Scaled by the material parameter of maximum hysteresis width $\beta$ it always points in the middle of each hysteresis. The driving force $\Delta \psi$

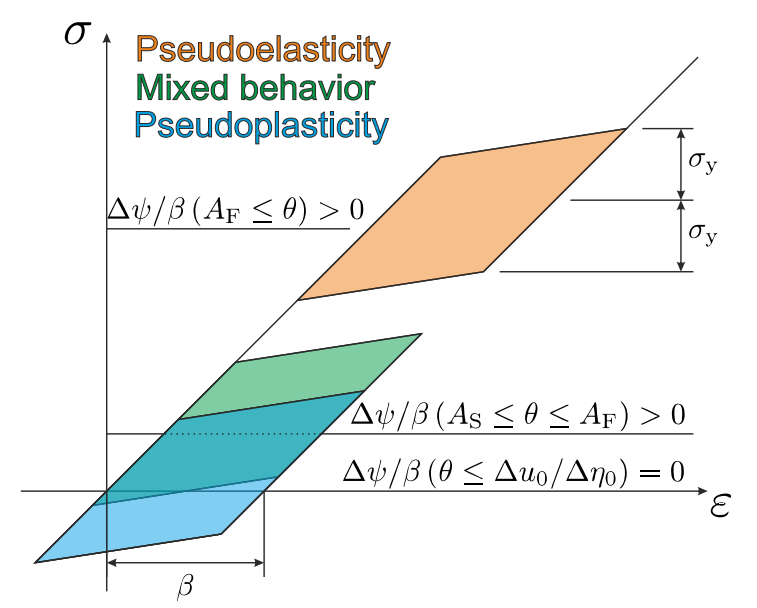

Figure 1. Off-set properties of $\Delta \psi$ and other material parameters

is the most important quantity for the transformation of the material from pseudoplasticity at low temperature to pseudoelasticity at the high temperature range, see Fig. 1. 


\subsection{Clausius Duhem Inequality}

The $2^{\text {nd }}$ law of thermodynamics is used in the form of the Clausius Duhem Inequality

$$
\mathcal{D}=\sigma \cdot \dot{\varepsilon}^{i}+p \cdot \dot{\alpha}-\Delta \psi \cdot \dot{z}_{/ /} \geq 0 \text {. }
$$

It can be seen easily that the stress $\sigma$ dissipates energy on the inelastic strain $\varepsilon^{i}$, the backstress $p$ works on the inner hardening variable $\alpha$ and the energy difference $\Delta \psi$ is a work conjugate variable to the oriented martensite $z_{/ /}$, see section 2.7 for a more detailed explanation. Equation (13) being positive indicates inelastic material behavior and elastic behavior being zero. Values less than zero are not admissible.

\subsection{Evolution Equations}

Evolution equations for the internal state variables $\varepsilon^{i}, \alpha, z_{/ /}$are derived via a Lagrangian functional. $\lambda$ is the Lagrangian parameter. In other words, we search the extrema of $\mathcal{D}$ under the constraint that the yield condition $\Phi=0$ [25] is fulfilled which reads

$$
\mathcal{L}=-\mathcal{D}+\lambda \cdot \Phi \rightarrow \text { stationary }
$$

To this optimization problem the following equation needs to be satisfied

$$
\nabla \mathcal{D}=\lambda \cdot \nabla \Phi
$$

According to the principle of maximum dissipation, we demand to which inner variables is $\mathcal{D}$ maximal under the constrained $\Phi=0$ and $\lambda>0$ so that the consistency condition $\lambda \cdot \Phi=0$ is fulfilled. Applying the nabla-operator on $\mathcal{D}$ and $\Phi$ yields

$$
\left[\begin{array}{c}
\frac{\partial \mathcal{D}}{\partial \Phi} \\
\frac{\partial \mathcal{D}}{\partial p} \\
\frac{\partial \mathcal{D}}{\partial \Delta \psi}
\end{array}\right]=\lambda \cdot\left[\begin{array}{c}
\frac{\partial \Phi}{\partial q} \\
\frac{\partial \Phi}{\partial p} \\
\frac{\partial \Phi}{\partial \Delta \psi}
\end{array}\right] .
$$

In equation (16) the phase transition equation $\Phi$ has to be replaced by $\vec{\Phi}$ in the case of loading or $\overleftarrow{\Phi}$ in the case of unloading. The same holds for the argument $\operatorname{sign}(\ldots)$ in the following equation

$$
\left[\begin{array}{c}
\dot{\varepsilon}^{i} \\
\dot{\alpha} \\
\dot{z}_{/ /}
\end{array}\right]=\lambda \cdot\left[\begin{array}{c}
+\operatorname{sign}\left(\sigma-p-\frac{\langle\Delta \psi\rangle}{\beta} \cdot \operatorname{sign}(\ldots)\right) \\
-\operatorname{sign}\left(\sigma-p-\frac{\langle\Delta \psi\rangle}{\beta} \cdot \operatorname{sign}(\ldots)\right) \\
\frac{1}{\beta} \cdot \operatorname{sign}\left(\sigma-p-\frac{\langle\Delta \psi\rangle}{\beta} \cdot \operatorname{sign}(\ldots)\right)
\end{array}\right] .
$$

Equation (17) represents all evolution equations to describe the optimization problem. Working with this complex of formulas guarantees the stationarity of equation (14).

\subsection{Martensite-Volume-Fraction}

The microstructure of shape memory alloys provides two different types of martensite. There is the twinned martensite $z_{\rangle}$which can be found in the unloaded material in the low temperature range. This martensite changes into austenite on heating. That is why literature 
often mentions it as temperature-induced martensite [12], [13]. The other type of martensite is an oriented martensite $z_{/ /}$which evolves on loading the material. Each of those martensite fractions may vary between zero and one. For the coexistence of both martensite fractions an additive split is chosen. This additive decomposition was introduced by [6] and is also used in [9], [12], [22].

$$
0 \leq z_{\rangle} \leq 1 ; \quad 0 \leq z_{/ /} \leq 1 ; \quad 0 \leq z \leq 1 ; \quad z=z_{/ /}+z_{\rangle\rangle}
$$

The evolution of the oriented martensite is given in equation (17) $)_{3}$. It can be integrated and reads then

$$
\dot{z}_{/ /}=\frac{\dot{\varepsilon}^{i}}{\beta} \Longrightarrow z_{/ /}=\frac{\varepsilon^{i}}{\beta} .
$$

It is demanded that $z_{/ /} \geq 0$. To ensure this, the evolution equation is manipulated as follows

$$
z_{/ /}=\frac{\left|\varepsilon^{i}\right|}{\beta}
$$

The evolution of the temperature-induced, twinned martensite is outlined in Fig. 2. The activation temperatures for the phases austenite $A$ and martensite $M$ are introduced. The indices $(\bullet)_{\mathrm{S}}$ and $(\bullet)_{\mathrm{F}}$ mark the start and the finish of the phase transformation. The corresponding

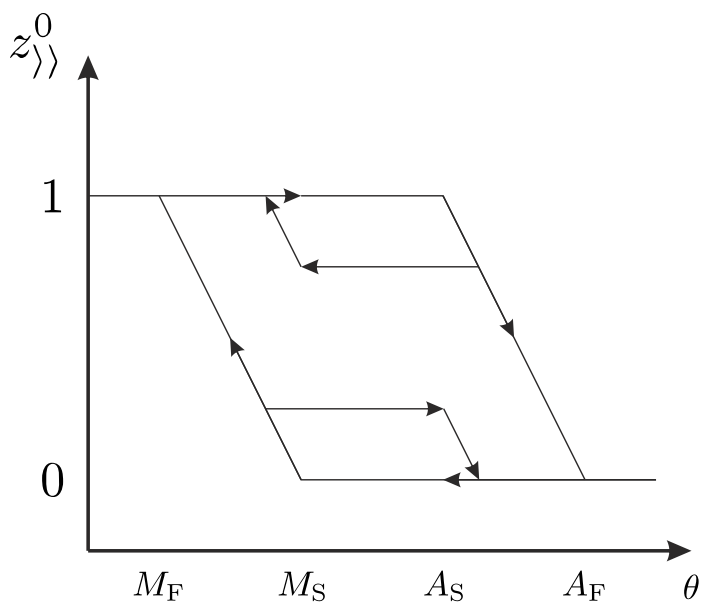

Figure 2. Evolution of temperature induced martensite

evolution equation reads

$$
\dot{z}_{>>}^{0}= \begin{cases}-\frac{\dot{\theta}}{A_{\mathrm{F}}-A_{\mathrm{S}}} & , \text { if } \dot{\theta}>0 \text { and } A_{\mathrm{S}}<\theta<A_{\mathrm{F}} \\ +\frac{M_{\mathrm{S}}-M_{\mathrm{F}}}{M} & , \text { if } \dot{\theta}<0 \text { and } M_{\mathrm{F}}<\theta<M_{\mathrm{S}} . \\ 0 & , \text { otherwise }\end{cases}
$$

Three different cases have to be considered: pseudoplasticity, an intermediate state and pseudoelasticity. First of all, there is the case of pseudoplasticity, Fig. 3. Here, the stress-strainbehavior forms a hysteresis around the origin of the coordinate system. During this process the martensite fraction changes from twinned martensite to oriented martensite. The volume fraction evolution of $z_{\rangle}$can be described as

$$
z_{\rangle\rangle}=1-z_{/ /}
$$


For pseudoelasticity the stress-strain-behavior shows hysteresises in the first and the third quadrant of the $\sigma$ - $\varepsilon$-diagram. This material behavior can be observed in the high temperature range. In the unloaded state no martensite exists. When the material is loaded oriented martensite evolves. Twinned martensite

$$
z\rangle\rangle=0
$$

is not present at any time, see Fig. 5. Between the austenite-start-temperature $A_{\mathrm{S}}$ and the austenite-finish-temperature $A_{\mathrm{F}}$ shape memory alloys transform from martensite to austenite due to temperature changes. Although, the model must guarantee that the material can be loaded at any time during this transformation. For this purpose the following ansatz

$$
z_{\rangle\rangle}\left(\varepsilon^{i}\right)=-\frac{z_{\eta\rangle}^{0}}{\beta} \cdot\left|\varepsilon^{i}\right|+z_{\rangle\rangle}^{0}
$$

is proposed, Fig. 4.

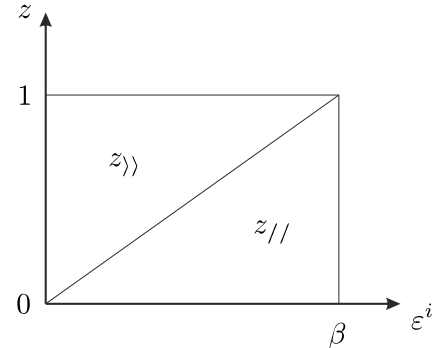

Figure 3. Pseudoplasticity

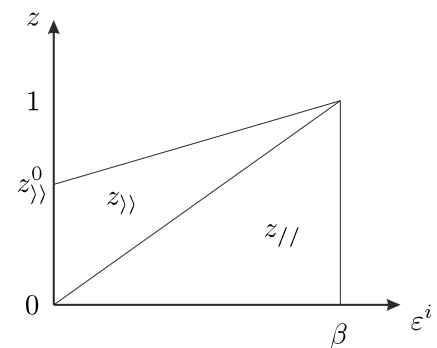

Figure 4. Intermediate state

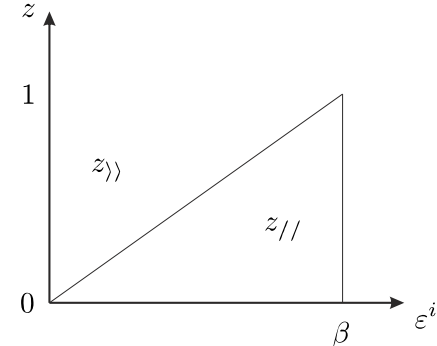

Figure 5. Pseudoelasticity

\subsection{Transformation Temperatures}

The characteristic transformation temperatures are dependent of the chosen material parameter set. The austenite-start-temperature marks the temperature to which a deformed structure starts the redeformation into its former shape (see Fig. 6). For the equilibrium state

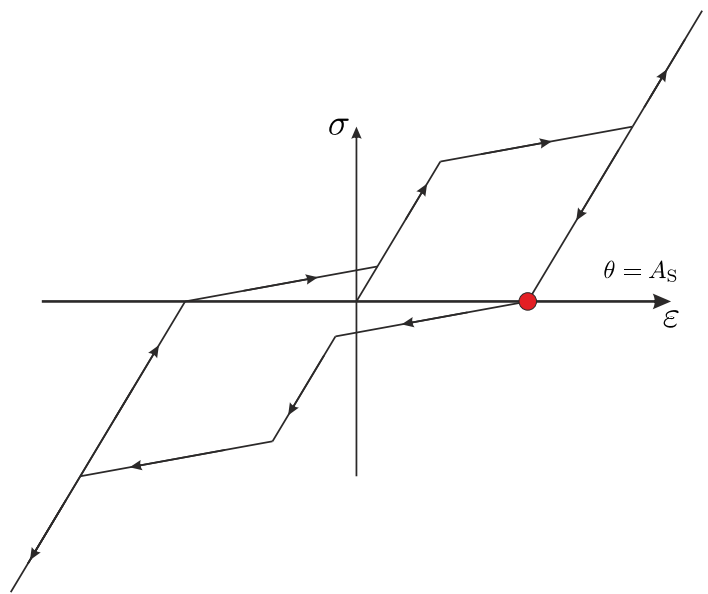

Figure 6. Austenite-start

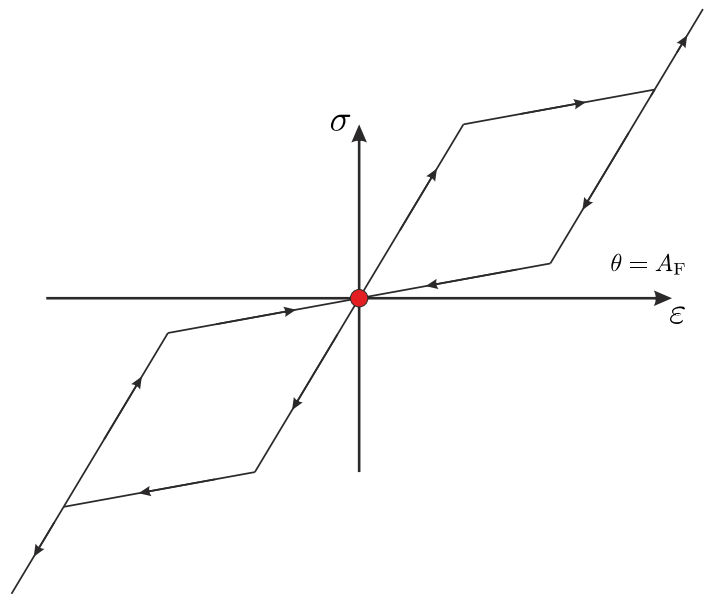

Figure 7. Austenite-finish 
marked in Fig. 6 with a red dot the phase transformation condition takes the following form

$$
\begin{aligned}
\overleftarrow{\Phi}(\sigma, p, \Delta \psi) & =\left|\sigma-p-\frac{\langle\Delta \psi\rangle}{\beta} \cdot \operatorname{sign}\left(\varepsilon^{i}\right)\right|-\sigma_{y}=0 \\
& =\left|0-K \cdot \beta-\frac{\left\langle\Delta u_{0}-A_{\mathrm{S}} \cdot \Delta \eta_{0}\right\rangle}{\beta} \cdot \operatorname{sign}(\beta)\right|-\sigma_{y}=0
\end{aligned}
$$

It follows the definition of the austenite-start-temperature $A_{\mathrm{S}}$ as

$$
A_{\mathrm{S}}=+\frac{\Delta u_{0}}{\Delta \eta_{0}}+\frac{\sigma_{y} \beta}{\left|\Delta \eta_{0}\right|}-\frac{K \beta^{2}}{\left|\Delta \eta_{0}\right|} .
$$

The austenite-finish-temperature (Fig. 7) is derived analogically by

$$
\begin{aligned}
\overleftarrow{\Phi}(\sigma, p, \Delta \psi) & =\left|\sigma-p-\frac{\langle\Delta \psi\rangle}{\beta} \cdot \operatorname{sign}\left(\varepsilon^{i}\right)\right|-\sigma_{y}=0 \\
& =\left|0-0-\frac{\left\langle\Delta u_{0}-A_{\mathrm{S}} \cdot \Delta \eta_{0}\right\rangle}{\beta} \cdot(+1)\right|-\sigma_{y}=0
\end{aligned}
$$

The term for the austenite-finish-temperature results in

$$
A_{\mathrm{F}}=+\frac{\Delta u_{0}}{\Delta \eta_{0}}+\frac{\sigma_{y} \beta}{\left|\Delta \eta_{0}\right|}
$$

To complete the set of transformation-temperatures the martensite-temperatures

$$
\begin{gathered}
M_{\mathrm{S}}=+\frac{\Delta u_{0}}{\Delta \eta_{0}}-\frac{\sigma_{y} \beta}{\left|\Delta \eta_{0}\right|} \\
M_{\mathrm{F}}=+\frac{\Delta u_{0}}{\Delta \eta_{0}}-\frac{\sigma_{y} \beta}{\left|\Delta \eta_{0}\right|}-\frac{K \beta^{2}}{\left|\Delta \eta_{0}\right|}
\end{gathered}
$$

may be derived from Fig. 8 [6], [19].

\section{Finite Element Implementation}

For numerical analysis the material model is embedded into the finite element method. Conforming to the 1-D material model a formulation for a truss element is chosen. The finite element implementation is derived on basis of the balance of linear momentum

$$
\left.\frac{\partial}{\partial x} \sigma+\rho b=\rho \frac{\partial v}{\partial t} \text { in } \mathcal{B} \times\right] 0, T[
$$

For the 1-D case, neglecting dynamic effects and own weight, eq. (31) simplifies to the following differential equation

$$
\frac{\partial N}{\partial x} \mathrm{~d} x+n(x) \cdot \mathrm{d} x \Rightarrow N^{\prime}+n=0
$$

with normal force $N$ in the infinitesimal beam element $\mathrm{d} x$ and axial load $n(x)$. The boundary conditions read

$$
N=\bar{N}=F \text { on } \partial_{\sigma} \mathcal{L}
$$




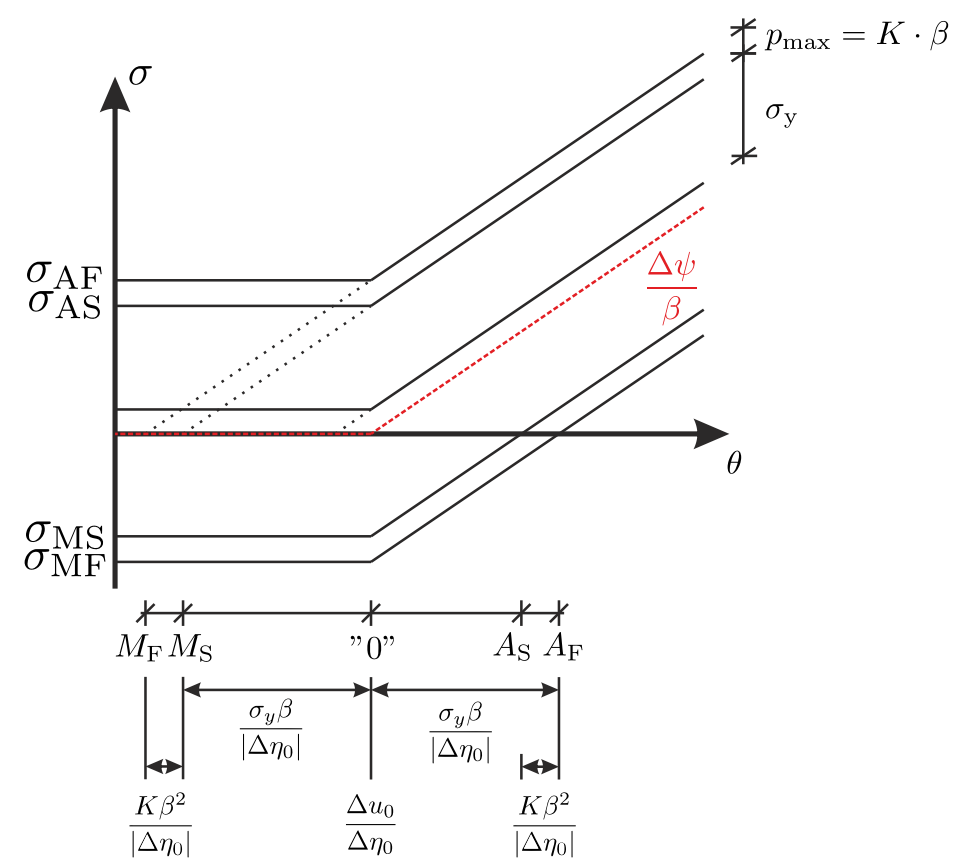

Figure 8. Stress-temperature-diagram for shape memory alloys (see [6])

on the loaded border of the element $\mathcal{L}$. Thus, the weak form of equilibrium can be written to

$$
g:=\int_{L} \delta \varepsilon N \mathrm{~d} x-\int_{L} \delta u \cdot n \mathrm{~d} x-F[\delta u]_{\partial_{\sigma} \mathcal{L}}=0 .
$$

The displacement is approximated by linear shape functions. On element level the approximated weak form reads

$$
\begin{aligned}
g_{e}^{\mathrm{h}} & =\int_{L_{e}} \delta \varepsilon^{\mathrm{h}} N \mathrm{~d} x-\delta u^{\mathrm{h}} \cdot n-F\left[\delta u^{\mathrm{h}}\right]_{\partial_{\sigma} \mathcal{L}_{e}} \\
& =\delta \boldsymbol{d}_{e}^{\mathrm{T}} \underbrace{\left(\int_{L_{\mathrm{e}}} \boldsymbol{B}^{\mathrm{T}} N \mathrm{~d} x\right.}_{\mathbf{f}_{e}^{\text {int }}}-\underbrace{\left.\int_{L_{\mathrm{e}}} \boldsymbol{N}^{\mathrm{T}} n \mathrm{~d} x-\left[\boldsymbol{N}^{\mathrm{T}} F\right]_{\partial_{\sigma} \mathcal{L}_{e}}\right)}_{\mathbf{f}_{e}^{\text {ext }}} .
\end{aligned}
$$

$\boldsymbol{N}$ contains the shape functions and the $\boldsymbol{B}$-matrix consists of the derivation of the shape functions. The displacement-vector $\boldsymbol{d}$ consists of the axial displacements of each node of the finite element. Since the virtual displacements $\delta \boldsymbol{d}_{e}^{\mathrm{T}}$ are arbitrary,

$$
\boldsymbol{G}=\bigcup_{e=1}^{\text {nelem }}\left(\mathbf{f}_{e}^{\mathrm{int}}-\mathbf{f}_{e}^{\mathrm{ext}}\right)=\mathbf{0}
$$

is in accordance with a global equilibrium condition. The nonlinear problem is linearized via a Taylor expansion.

$$
\boldsymbol{G}\left(\boldsymbol{u}^{k+1}\right)=\boldsymbol{G}\left(\boldsymbol{u}^{k}\right)+\frac{\partial \boldsymbol{G}\left(u^{k}\right)}{\partial \boldsymbol{d}^{k}} \cdot \Delta \boldsymbol{d}^{k}=\mathbf{0}
$$

Thus, $\boldsymbol{G}\left(\boldsymbol{u}^{k}\right)$ is the residual and the element stiffness matrix can be written to

$$
\boldsymbol{K}_{\mathrm{T} e}=\frac{\partial \boldsymbol{G}_{e}}{\partial \boldsymbol{d}_{e}^{k}}=\int_{L_{e}} \boldsymbol{B}^{\mathrm{T}} \cdot A \cdot \mathbb{C}_{\mathrm{T}} \cdot \boldsymbol{B} \mathrm{d} x .
$$


$\mathbb{C}_{\mathrm{T}}$ denotes the consistent tangent modulus given as

$$
\mathbb{C}_{\mathrm{T}}=\frac{\partial \sigma^{k}}{\partial \varepsilon^{k}}=\left\{\begin{array}{ll}
E & : \text { elastic step } \\
\frac{E K}{E+K} & : \text { inelastic step }
\end{array} .\right.
$$

\section{Numerical Examples}

Two numerical examples show the capability of the presented model. Both examples are modeled with the same set of material parameters (table 1) for the shape memory alloy.

Table 1. Material parameters for shape memory alloy [8]

\begin{tabular}{lccr}
\hline Material parameter & Abbreviation & Unit & Value \\
\hline Young's modulus & $E$ & $\mathrm{MPa}$ & 60000.00 \\
Hardening modulus & $K$ & $\mathrm{MPa}$ & 2000.00 \\
Half height of hysteresis & $\sigma_{\mathrm{y}}$ & $\mathrm{MPa}$ & 120.00 \\
Maximum hysteresis width & $\beta$ & {$[-]$} & 0.05 \\
Difference of inner energy & $\Delta u_{0}$ & $\mathrm{MPa}$ & -204.00 \\
Difference of entropy & $\Delta \eta_{0}$ & $\mathrm{MPa} /{ }^{\circ} \mathrm{K}$ & -0.80 \\
\hline Martensite-finish & $M_{\mathrm{F}}$ & ${ }^{\circ} \mathrm{K}$ & 241.25 \\
Martensite-start & $M_{\mathrm{S}}$ & ${ }^{\circ} \mathrm{K}$ & 247.50 \\
Austenite-start & $A_{\mathrm{S}}$ & ${ }^{\circ} \mathrm{K}$ & 256.25 \\
Austenite-finish & $A_{\mathrm{F}}$ & ${ }^{\circ} \mathrm{K}$ & 262.50 \\
\hline
\end{tabular}

\subsection{1-D-Rod}

For a 1-D rod the following five loading situations are analyzed. Examples 1 and 2 are calculated with an external, axial load. The load is raised with a proportional load factor at first und decreased subsequently. Pseudoplastic and pseudoelastic behavior can be observed. For examples 3-5 a prestrained $\left(\varepsilon=\varepsilon^{i}=\beta\right)$ rod is considered and the temperature is varied. The different shape memory effects take place. All results are displayed in Figs. 9 and 10.

\section{Pseudoplasticity and Shape Memory Effect}

A surrounding temperature of $\theta=250{ }^{\circ} \mathrm{K}$ is assumed. The driving force $\Delta \psi$ is zero.

Reaching the initial yield-stress $\sigma_{\mathrm{y}}=120 \mathrm{MPa}$ the martensite-twins are starting to orientate (dotted, blue line). At an inelastic strain $\varepsilon^{i}=\beta=0.05$ linear elastic material behavior is observed again.

Unloading subsequently, a remnant strain of $\varepsilon=\beta=0.05$ remains. When the structure is heated above $A_{\mathrm{S}}=256.25{ }^{\circ} \mathrm{K}$ the shape memory effect is initiated and the strain decreases again, see Fig. 9. At a temperature $\theta=A_{\mathrm{F}}=262.50{ }^{\circ} \mathrm{K}$ the structure is completely in its original shape. The microstructure is austenitic. A subsequent cooldown below $M_{\mathrm{F}}=241.25^{\circ} \mathrm{K}$ recovers the twinned martensite.

2. Pseudoelasticity at $\theta=300^{\circ} \mathrm{K}$.

The driving force measures $\Delta \psi=\Delta u_{0}-\theta \cdot \Delta \eta=36 \mathrm{MPa}$ at this temperature. The hysteresis is shifted into the first quadrant (dashed, green line). With $\sigma=\Delta \psi / \beta+\sigma_{\mathrm{y}}=$ 
$840 \mathrm{MPa}$ the austenite transforms into oriented martensite. At hysteresis width $\beta=0.05$ elastic material behavior can be observed again. The material has completely transformed into oriented martensite (stress-induced). Unloading the material lets the material accommodate in the origin of the stress-strain-diagram. All strains and stresses are zero. The material is completely austenitic again.

Each of the following calculations start at an initial temperature $\theta=250{ }^{\circ} \mathrm{K}$. At this temperature a material behavior like in point 1 . occurs. In contrast to the examples above the shape memory effect is constrained by a spring at the tip of the rod.

3. Two-Way Shape Memory Effect-Case I.

Here, a spring with a stiffness of $E A / L=1 \cdot 10^{4} \mathrm{MN} / \mathrm{m}$ is placed at the tip of the rod. Reaching the austenite-start-temperature $A_{\mathrm{S}}$ the shape memory effect begins. A transformation back into the former state is constrained by the spring. The spring induces stresses into the rod and while the temperature rises pseudoelastic material response evolves and high stresses $\sigma=428.54 \mathrm{MPa}$ are achieved (dash dotted, black line). No matter how high the temperature is risen the strains cannot be driven back to zero completely $(\varepsilon=0.0071457)$. When the rod is cooled down the stress decreases and the strain increases again. Finally, there is a remnant stress of $\sigma=166.43 \mathrm{MPa}$ (Fig. 10) and a remnant strain $\varepsilon=0.033357$. This procedure is repeatable.

\section{Two-Way Shape Memory Effect-Case II.}

This time, a spring with a stiffness of $E A / L=1 \cdot 10^{5} \mathrm{MN} / \mathrm{m}$ is used. The behavior is similar to the one described before. Now, even higher stresses $\sigma=1874.50 \mathrm{MPa}$ are admissible (dash dotted, turquoise line). But the minimal strain $\varepsilon=0.031255$ is a bit greater. Cooling down the rod leads to a residual stress $\sigma=194.87 \mathrm{MPa}$ (Fig. 10) and strain $\varepsilon=0.048051$.

\section{Constrained Shape Memory Effect.}

In the last calculation, the redeformation is completely constrained. The stiffness of the spring is infinite. Thus, the rod is clamped. That's the reason why it cannot recover strain and just the stresses rise up to stresses greater than $\sigma=2000.00 \mathrm{MPa}$ (dotted, pink line). After a cool-down, there is a stress remaining which values $\sigma=199.00 \mathrm{MPa}$ (Fig. 10).

\subsection{Grip-Element}

In this section an example is presented which shows the capability of the formulation to interact with other elements and materials. A grip-element is modeled which consists of three arms. They are discretized with eight-node-brick-elements. Each arm has a length of $L=180 \mathrm{~mm}$ and a cross-section $b \times h=30 \times 30 \mathrm{~mm}^{2}$. They consist of $9 \times 3 \times 3$ elements, see Fig. 11. The grip-elements are modeled by a soft rubber-like material with a young's modulus $E=100 \mathrm{MPa}$ and a Poisson ratio $\nu=0$. Beneath every arm, there are four rows of shape memory alloy fibers in axial direction, see Fig. 11. They are prestrained to their maximum hysteresis width $\varepsilon^{i}=\beta=0.05$. 

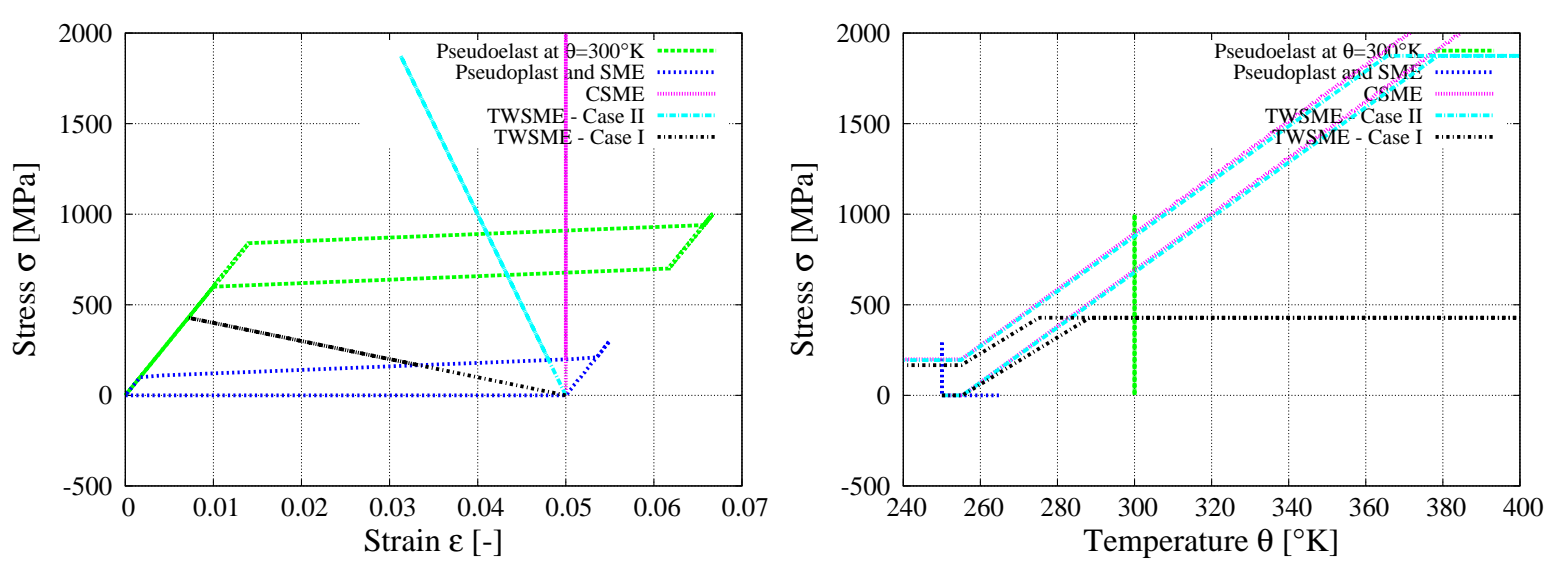

Figure 9. Stress-strain-diagram for a 1-D- Figure 10. Stress-temperature-diagram for a rod at different temperatures $1-\mathrm{D}-\mathrm{rod}$

When the structure is heated and the austenite-finish-temperature is reached the shape memory alloys begin to shrink. Due to the excentric position of the fibers the grip elements start to bend, see Fig. 12, Fig. 14. The maximum deflection is reached at a temperature $\theta_{\text {cf }}=282{ }^{\circ} \mathrm{K}$ and amounts $w_{\mathrm{cf}}=35 \mathrm{~mm}$. The vertical deflection of the upper arm is measured. When the temperature decreases a redeformation starts at $\theta_{\text {os }}=266^{\circ} \mathrm{K}$. A two-way shape memory effect can be observed which is comparable to section 4.1 case no. 3 and 4 . The grip-elements open up to a deflection of $w_{\text {of }}=20 \mathrm{~mm}$ at a temperature $\theta_{\text {of }}=256{ }^{\circ} \mathrm{K}$, see Fig. 13. From now on, the process is repeatable. Between the temperature range $\theta_{\text {of }}$ and $\theta_{\text {cf }}$ all deflections between $w_{\text {of }}$ and $w_{\text {cf }}$ are admissible, compare Fig. 14.

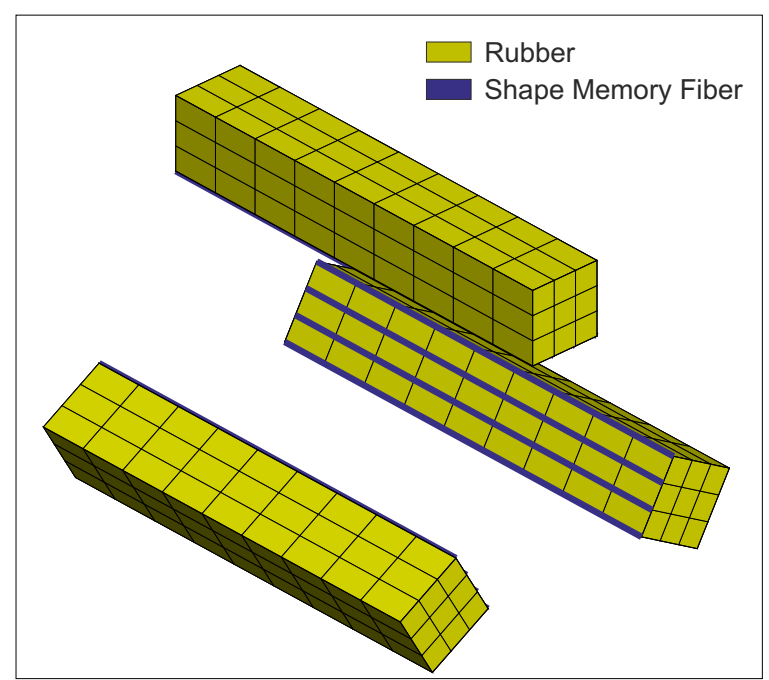

Figure 11. Undeformed grip-element $\theta=240{ }^{\circ} \mathrm{K}$

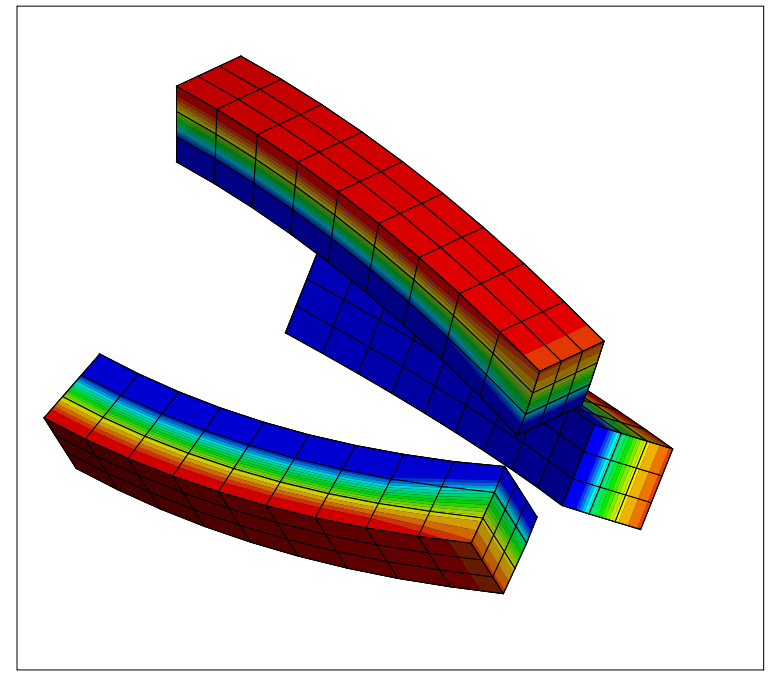

Figure 12. Maximum deformation grip-element, $\theta=282^{\circ} \mathrm{K}$

\section{Summary and Outlook}

This contribution deals with an efficient and fast algorithm for shape memory fibers. All important material phenomena like pseudoplasticity, pseudoelasticity, the free, two-way 


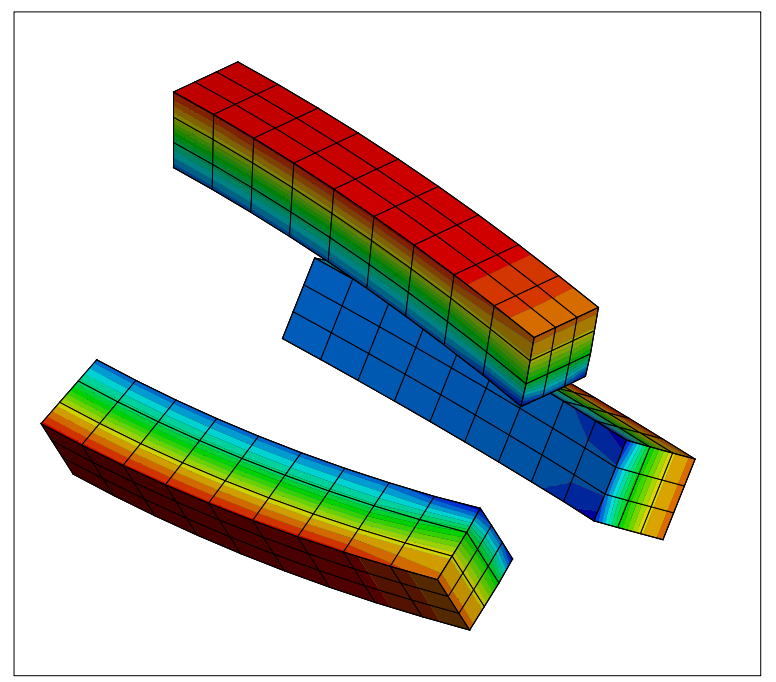

Figure 13. Residual deformation grip-element, $\theta=256^{\circ} \mathrm{K}$

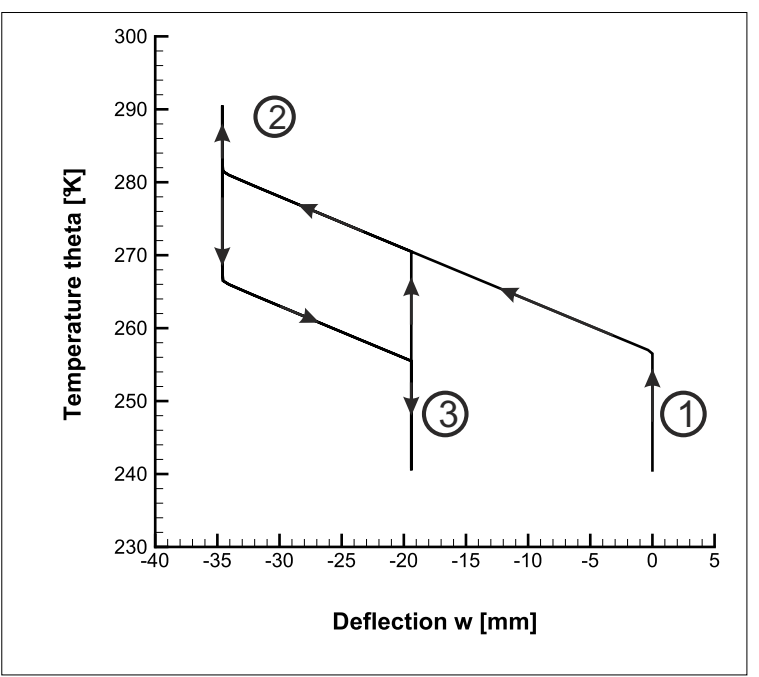

Figure 14. Temperature-deflection-diagram grip-element

and the constrained shape memory effect are captured. The 1-D formulation allows for easy meshing in the framework of the finite element method. No problems are encountered combining the shape memory - truss formulation with other structures or materials.

It is possible to use the formulation to model and analyze reinforced structures. The present model puts emphasis on the proper simulation of the phase transition from martensite to austenite no matter which loading conditions are applied. This allows for the ability to prestress a surrounding matrix and amend brittle material. It should be possible to predict the material behavior of complex compound-materials of shape memory fiber in combination with any other material.

The simulation of greater structures would ask for an enormous number of elements to represent the microstructure of fiber-composites. Here, a multiscale-approach is conceivable. By the use of a coarse mesh on the macroscale and a representative volume element (RVE) on the microscale, information can be passed between them both and calculation-time can be spared eventually.

\section{REFERENCES}

[1] Achenbach, M., Müller, I., "A Model for Shape Memory”. J. Phys. Colloques, Vol. 43, No. C4, 163-167, 1982.

[2] Bo, Z., Lagoudas, D., "Thermomechanical modeling of polycrystaline SMAs under cyclic loading, Part I: theoretical derivations". International Journal of Engineering Science, 37, 1089-1140.

[3] Bo, Z., Lagoudas, D., “Thermomechanical modeling of polycrystaline SMAs under cyclic loading, Part II: material characterization and experimental results for a stable transformation cycle". International Journal of Engineering Science, 37, 1141-1173. 
[4] Bo, Z., Lagoudas, D., "Thermomechanical modeling of polycrystaline SMAs under cyclic loading, Part III: evolution of plastic strains and two-way shape memory effect". International Journal of Engineering Science, 37, 1175-1203.

[5] Bo, Z., Lagoudas, D., "Thermomechanical modeling of polycrystaline SMAs under cyclic loading, Part IV: modeling of minor hysteresis loops". International Journal of Engineering Science, 37, 1205-1249.

[6] Brinson, L. C., "One-Dimensional Constitutive Behavior of Shape Memory Alloys: Thermomechanical Derivation with Non-Constant Material Functions and Redefined Martensite Internal Variable". Journal of Intelligent Material Systems and Structures, 4, 229-242, 1993.

[7] Castellana, M., Colato, G., Infanti, S., Use of Viscous Dampers and Shock Transmission Units in the Seismic Protection of Buildings". 13th World Conference on Earthquake Engineering, Vancouver, 2004.

[8] Christ, D., Reese, S., "Thermomechanically coupled modelling of shape memory alloys in the framework of large strains". GAMM-Mitt. 31, No. 2, 176-191, 2008.

[9] Christ, D., Reese, S., "A finite element model for shape memory alloys considering thermomechanical couplings at large strains". Int. J. of Solids and Structures , 46, 3694-3709, 2009.

[10] Christ, D., "Thermomechanical modelling of shape memory alloy structures in medical applications". Ph.D. thesis, Institut für Festkörpermechanik, TU Braunschweig, 2009.

[11] Evangelista, V., Marfia, S., Sacco, E., "Phenomenological 3D and 1D consistent models for shape-memory alloy materials". Comput. Mech. 44, 405-421, 2009.

[12] Helm, D., "Formgedächtnislegierungen: Experimentelle Untersuchung, phänomenologische Modellierung und numerische Simulation der thermomechanischen Materialeigenschaften". Ph.D. thesis, Institut für Mechanik, Universität Gesamthochschule Kassel, Kassel, 2001.

[13] Helm, D., Haupt, P., "Shape memory behavior: modelling within continuum thermomechanics". Int. J. of Solids and Structures , 40, 827-849, 2003.

[14] Helm, D., "Thermomechanics of martensitic phase transitions in shape memory alloys - constitutive theories for small and large deformations". Journal of Mechanics of Materials and Structures, Vol. 2, No. 1, 87-112, 2007.

[15] Helm, D., Haupt, P., "Numerical simulation of martensitic phase transitions in shape memory alloys using an improved integration algorithm”. Int. J. Numer. Meth. Engng, 69, 1997-2035, 2007.

[16] Indirli, M., Castellano, M.G., Clemente, P., Martelli, A., "Demo-Application of Shape Memory Alloy Devices: the Rehabilitation of the S. Giorgio Church Bell Tower". Proc. SPIE Smart Systems for Bridges, Structures, and Highways Invited paper n. 4330-30, , Newport Beach, California, USA, 2001.

[17] Indirli, M., Castellano, M.G., "Shape Memory Alloy Devices for the structural improvement of masonry heritage structures". International Journal of Architectural Heritage, Conservation, Analysis and Restoration Volume 2, Number 2, 93-119, 2008.

[18] Janke, L., Czaderski, C., Motavalli, M., Ruth, J., "Applications of Shape Memory Alloys in Civil Engineering Structures”. Materials and Structures, 279, 578-592, 2005. 
[19] Juhász, L., "Herleitung eines konstitutiven Modells für Formgedächtnislegierungen". Ph.D. thesis, Institut für Technische Mechanik, Universität Karlsruhe (TH), Karlsruhe, 2004.

[20] Klinkel, S., Kohlhaas, B., "Modellierung und Anwendung von Formgedächtnislegierungen im Bauwesen”, Bauingenieur, Jahresausgabe 2011/2012, Springer VDI Verlag, 101-110, 2011.

[21] Lagoudas, D., "Shape Memory Alloys - Modeling and Engineering Applications". Springer, 2008.

[22] Leclercq, S., Lexcellent, C., "A General Macroscopic Description of the Thermomechanical Behavior of Shape Memory Alloys”. J. Mech. Phys. Solids, Vol. 44, No. 6, 953-980, 1996.

[23] McCormick, J., DesRoches, R., Fugazza, D., Auricchio, F., "Seismic Vibration Control Using Superelastic Shape Memory Alloys". Transactions of the ASME, 128, 294-301, 2006.

[24] Moser, K., Bergamini, A., Christen, R., Czaderski, C.: "Feasibility of concrete prestressed by shape memory alloy short fibres". Materials and Structures, 38, 593-600, 2005.

[25] Neunzert, H., Eschmann, W. G., Blickensdörfer-Ehlers, A., Schelkes, K., "Analysis 2. With an introduction to vector and matrix calculus. A text- and workbook". SpringerLehrbuch, 1980.

[26] Raniecki, B., Lexcellent, C., "Thermodynamics of isotropic pseudoelasticity in shape memory alloys". European Journal of Mechanics - A/Solids, 17 (2), 185-205, 1998.

[27] Seelecke, S., Müller, I., "Shape memory alloy actuators in smart structures: Modeling and simulation". Applied Mechanics Reviews, 57 (1), 23-46, 2004.

[28] Simo, J. C., Hughes, T. J. R., “Computational Inelasticity”. Interdisciplinary Applied Mathematics, 7, 1998.

[29] Song, G., Ma, N., Li, H.-N., "Applications of shape memory alloy in civil structures". Engineering Structures, 28, 1266-1274, 2006. 